\title{
BENEFITS OF USING CONDUCTIVE PLASTICS IN SHIELDING CONFIGURATIONS TO REDUCE RADIATED ELECTROMAGNETIC INTERFERENCE
}

\begin{tabular}{|r|l|}
\hline Journal: & Microwave and Optical Technology Letters \\
\hline Manuscript ID: & MOP-10-0101 \\
\hline Wiley - Manuscript type: & Research Article \\
\hline Date Submitted by the \\
Author: & 26 -Jan-2010 \\
\hline Complete List of Authors: & $\begin{array}{l}\text { Lozano-Guerrero, Antonio; Universidad Politécnica de Cartagena, } \\
\text { Departamento de Tecnologías de la Información y las } \\
\text { Comunicaciones } \\
\text { Robinson, Martin; The University of York, Department of Electronics } \\
\text { Diaz-Morcillo, Alejandro; Polytechnic University of Cartagena, } \\
\text { Information Technologies and Communicactions } \\
\text { Balbastre-Tejedor, Juan; Polytechnic University of Valencia, } \\
\text { Communications }\end{array}$ \\
\hline Keywords: & $\begin{array}{l}\text { conductive plastic, enclosure, Q-factor, radiated immunity, shielding } \\
\text { effectiveness }\end{array}$ \\
\hline \hline
\end{tabular}

\section{s ScholarONE" \\ Manuscript Central}




\title{
BENEFITS OF USING CONDUCTIVE PLASTICS IN SHIELDING CONFIGURATIONS TO REDUCE RADIATED ELECTROMAGNETIC INTERFERENCE
}

\author{
Antonio José Lozano-Guerrero, ${ }^{1}$ Martin Paul Robinson, ${ }^{2}$ Alejandro Díaz-Morcillo, ${ }^{1}$ and \\ Juan Vicente Balbastre-Tejedor ${ }^{3}$ \\ ${ }^{1}$ Departamento de Tecnologías de la Información y las Comunicaciones \\ Universidad Politécnica de Cartagena \\ Cartagena, Spain \\ ${ }^{2}$ Department of Electronics \\ University of York \\ York, UK \\ ${ }^{3}$ Instituto ITACA \\ Universidad Politécnica de Valencia \\ Valencia, Spain \\ email: antonio.lozano@upct.es
}

Fax: +34 968325973

\begin{abstract}
Conductive plastic cabinets have become an alternative to traditional metallic enclosures to shield electronic equipment from electromagnetic interference. These materials allow a wide range of conductivities that can satisfy any particular design. In this paper the benefits of using conductive plastics in enclosure configurations have been evaluated. A design with an outer metallic layer and an inner layer of conductive dielectric can provide advantages from both materials since a conductive plastic box is lighter and its shielding properties may have advantages over metallic materials. An optimum for resonance suppression has been obtained for the hybrid structure. These shielding structures have been evaluated with the help of measurements and simulations. Shielding effectiveness and Q-factor have been used to compare the capabilities of these enclosures with the metallic ones showing their benefits and possibilities. Resonance suppression and shielding levels provided by conductive plastics are discussed.
\end{abstract}

Key words: Conductive plastic; enclosure; Q-factor; radiated immunity; shielding effectiveness 


\section{INTRODUCTION}

The use of conductive plastics for shielding purposes has become very common nowadays. Enclosures to protect electronic devices from unwanted electromagnetic radiation are being manufactured with plastic materials, which are cheaper and lighter than metals. However little has been researched regarding the study of their shielding properties and possibilities in enclosure configurations. A wide range of available conductivities may help to design cabinets with different requirements. Specific problems such as the minima in the shielding effectiveness (SE) curve related to the physical dimensions of the cabinet may be minimized with an optimized structure. The study of shielding effectiveness of metallic enclosures has been widely analysed through different approaches. Analytical solutions have been provided [1-2]. Numerical methods have also been necessary when enclosures with non-canonical shapes, arbitrary distribution of surface apertures and internal contents in the box have been analysed. Transmission Line Matrix (TLM) [3], Finite Difference Time Domain (FDTD) [4], and Method of Moments (MoM) [5] are some examples of the methods used.

The effect of resonance suppression is studied analytically in [6] for a non-realistic double spherical shell with no apertures in its surface. In [7] a study to suppress resonances with pieces of conductive polymers is carried out numerically with the Finite Element Method (FEM) including a PCB model but without optimising the polymer conductivity values. The use of resistive sheets applied near the apertures to reduce electromagnetic penetration is carried out in [8] but the influence of the enclosure is not taken into account.

In this paper two configurations including conductive plastics have been evaluated: (i) a hybrid solution with an outer metallic layer and an inner conductive dielectric layer as depicted in Figure 1 and (ii) a conductive dielectric enclosure.

\section{THEORY}

The classical definition of shielding effectiveness has been used to quantify the benefits of conductive plastics in shielding enclosure configurations. It is defined as the ratio of the field $\vec{E}_{i}$ obtained in the selected position in the absence of the shield and the field $\vec{E}_{t}$ at the same location with the shielding enclosure. It is usually expressed in logarithmic units

$$
\mathrm{SE}(\mathrm{dB})=20 \log _{10}\left(\frac{\left|\vec{E}_{i}\right|}{\left|\vec{E}_{t}\right|}\right) .
$$

The skin depth parameter also has special interest as the design of the inner conductive plastic layer follows the criterion established in [6] and [9], where the optimum conductivity to suppress a selected resonance verifies the ratio $\frac{t}{\delta} \approx 1.15$. It is a parameter that shows the penetration level of a wave in a medium and it is defined for the general case as 


$$
\delta=\frac{1}{\omega \sqrt{\frac{\mu \varepsilon}{2}\left(\sqrt{1+\left(\frac{\sigma}{\omega \varepsilon}\right)^{2}-1}\right)}} .
$$

where $\varepsilon=\varepsilon_{r}^{\prime} \varepsilon_{0}, \mu \approx \mu_{0}, \omega=2 \pi f, \sigma$ is the conductivity, $\varepsilon_{r}^{\prime}$ is the dielectric constant, $f$ the frequency under study and $\varepsilon_{0}$ and $\mu_{0}$ are the vacuum permittivity and permeability. For conductors $(\sigma>>\omega \varepsilon)$ the well-known approximation (3) can be applied

$$
\delta=\sqrt{\frac{2}{\omega \sigma \mu}} .
$$

Metallic materials have conductivities that lead to low values of skin depth compared to the thickness of any practical metallic enclosure at radio frequencies. Reducing the conductivity will decrease the shielding capabilities of an enclosure, but taking into account that shielding enclosures for electronic equipments have apertures on its surface for the input/output interfaces and ventilation requirements, the shielding capabilities of the cabinets are drastically reduced. In these applications, 30 or $40 \mathrm{~dB}$ can be considered a good protection level.

\section{SET UP}

The enclosure used for measurements and simulations is a $30 \times 12 \times 30 \mathrm{~cm}^{3}$ box. A $10 \mathrm{x}$ $0.5 \mathrm{~cm}^{2}$ aperture in the centre of the front face allows the coupling of energy from the outer to the inner part of the cabinet. For the conductive plastic study a four layer structure has been designed with sheets made from polystyrene plus carbon filler [10]. The reason for its use was its commercial availability. This structure fits exactly inside the surface of the box with a $0.3+0.3+0.1+0.1 \mathrm{~cm}$ sequence of layers. Discontinuities owing to air gaps between layers that could affect the measurements have been carefully avoided. By selecting different number of sheets, four equivalent inner layers with 0.8 , $0.7,0.6$, and $0.3 \mathrm{~cm}$ thicknesses can be then evaluated. Figure 2 shows the inner plastic structure with the outer metallic enclosure.

Measurements were carried out in an anechoic chamber. A log-periodic antenna in the range $30 \mathrm{MHz}-2000 \mathrm{MHz}$ was used as source. The antenna was placed $3 \mathrm{~m}$ away from the front panel of the box. A $4 \mathrm{~cm}$ long receiving monopole was placed in the geometric centre of the enclosure on the bottom wall inside the box. The reference value for the shielding effectiveness measurement was taken with the metallic plate containing the monopole. The shield was then added to the structure to measure the transmitted field inside the box.

Simulations have been carried out with CST Microwave Studio [11] commercial software (Finite Integration Technique).

To characterise the conductive plastic for the frequency range under study (30-2000 $\mathrm{MHz}$ ) measurements with an impedance/material analyser (Agilent HP4291A RF) have been carried out. Results for the central frequency $(1 \mathrm{GHz})$ have been selected to model the plastic structure $\left(\varepsilon_{r}^{\prime}=15\right.$ and $\sigma=0.39 \mathrm{~S} / \mathrm{m}$ ). Although this equipment is a low loss component analyser this conductivity value is within the limits given by the manufacturer and has been checked inversely by comparing simulations and 
measurements. Since this material is not intended for electromagnetic shielding purposes, thickness has been increased to provide reasonable resonance suppression.

\section{RESULTS}

Figure 3 shows the influence of increasing the thickness of the inner plastic coating experimentally for an empty metallic enclosure without electronic contents. Increasing the quantity of lossy material leads to increase the protecting properties of the shield as the coupling energy can be easily dissipated. Resonances have been dampened and shielding levels generally increase for the whole band under study. Table 1 shows the values of Q-factor obtained for the two first resonances. To obtain the Q-factor finely the procedure shown in [12] has been used. Influence of the thickness of the material can be clearly seen as for both resonances the Q-factor decreases monotonically with $t$ and $f 1$ and $f 2$ also decrease monotonically as a consequence of the dielectric loading.

In Figure 4 simulated and measured values for the hybrid structure with a $0.8 \mathrm{~cm}$ plastic layer have been depicted. Gap discontinuities or the simplicity of the used model (a constant conductivity and dielectric constant in a $2 \mathrm{GHz}$ band) may explain the little differences between simulation and measurements. Taking this into account the proposed equivalent to model the inner layer works fine for the hybrid structure.

Conductivity values of this material are not optimized for resonance suppression. If a conductive plastic of $0.2 \mathrm{~cm}$, a reasonable and practical thickness for a shielding enclosure is simulated for its optimum performance at $700 \mathrm{MHz}$ following the criterion in [6] $\left(\frac{t}{\delta} \approx 1.15\right)$ an approximate value of $\sigma=120 \mathrm{~S} / \mathrm{m}$ is obtained. Assuming a good conductor behaviour for the optimum conductivity at the frequency under study, the independence of the optimum conductivity with the dielectric constant [6] can be verified since the skin depth does not depend on this parameter. A value of $\varepsilon_{r}^{\prime}=2$ has been selected for the simulations.

In Figure 5 a sweep of conductivity values has been carried out for a $0.2 \mathrm{~cm}$ inner coating and a fixed $\varepsilon_{r}^{\prime}=2$ value. The second resonance SE values have been obtained $7.5 \mathrm{~cm}$ from the back wall near one of the two maxima of the electromagnetic mode. As expected the optimum values of SE for the first and second resonance follow the criterion in [6] caused by the maximization of reflection loss of the internal layer. This verifies the validity of the criterion in [6] when the shielding structure presents apertures on its surface. The curve also provides useful information about good design in this type of structure. After a stage of increasing resonance suppression towards the optimum conductivity, a slow fall is observed. Q values have been obtained for this sweep showing a similar behaviour in Figure 6.

Figure 7 shows the optimized results $(f=700 \mathrm{MHz}$ and $\sigma=120 \mathrm{~S} / \mathrm{m})$ for the hybrid (outer metallic layer + inner plastic coating) and plastic structure for a $0.2 \mathrm{~cm}$ layer. The metallic protection in a hybrid enclosure can be obtained by different ways: a metallic painting or a metallic enclosure could be used. In the case of a painting, scratches could decrease the shielding properties of the shield. If the metallic layer is removed results can be seen for the conductive dielectric structure. The $\mathrm{Q}$ factors for the first resonance are respectively 32.88 and 27.74 , showing a slight improvement in the conductive plastic enclosure. Figure 3 and Table I show similar benefits for the case of $0.8 \mathrm{~cm}$ commercial sample coating. The main difference is the shielding level obtained for the lower frequencies, although values higher than $30 \mathrm{~dB}$ provide good protection. If 
needed, higher conductivities may provide better SE levels depending upon the design requirements.

Although results have been obtained for the enclosure without any electronic contents similar results can be expected when the enclosure is loaded due to additive properties of Q factor.

Optimum designs will vary depending upon the suppression frequency. Higher frequencies will lead towards lower conductivities, decreasing the shielding levels for lower frequencies. Higher conductivities will increase shielding levels at lower frequencies but resonance suppression may be not as good as the optimum value. It must be pointed out that results obtained for the hybrid solution may not be optimum for the conductive dielectric case.

\section{CONCLUSION}

The benefits and possibilities of conductive plastics have been evaluated. Simulations and measurements have been carried out to compare the shielding effectiveness levels of conductive plastic structures with those of traditional metallic cabinets. The two main advantages of these structures are the resonance suppression provided for the resonance minima associated with the dimensions of the enclosure and the general increase of shielding levels at higher frequencies. Both aspects have been verified experimentally and numerically by obtaining SE curves and evaluating the Q-factor of the resonances. A sweep of conductivity values has been carried out to obtain the resonance suppression behaviour of an inner layer coating.

Two alternative enclosure configurations against radiated interference have been evaluated. Both solutions, a hybrid enclosure (metallic layer + conductive dielectric layer), and just a conductive dielectric offer similar results, which increase the shielding capabilities of the traditional metallic enclosures reducing the levels of interference inside the enclosure.

The hybrid solution can be optimized for one specific frequency to suppress the SE minima associated with enclosure resonances with apertures. It allows the good shielding behaviour of metallic enclosures at frequencies lower than the first resonance of the structure and a SE curve without abrupt minima due to resonance dampening produced by the inner lossy layer. Concerning the conductive plastic enclosure, shielding levels at lower frequencies are limited by the conductivity of the material, and shielding values at higher frequencies tend to the metallic ones as the skin depth value decreases with the frequency. According to the needs of a particular device, a specific design may be obtained nowadays since a wide range of conductivities can be obtained with conductive plastics.

\section{ACKNOWLEDGEMENTS}

This work was funded by Fundación Séneca, Agencia Regional de Ciencia y Tecnología, through the project 00700/PPC/04 and partly done during a research stay funded by the "Programa de formación y movilidad del personal docente e investigador de la Universidad Politécnica de Cartagena (PMPDI-UPCT-2007)" at the Physical Layer Group, Department of Electronics, University of York. 


\section{REFERENCES}

1. M. P. Robinson, T. M. Benson, C. Christopoulos, J. F. Dawson, M. C. Ganley, A. C. Marvin, S. J. Porter, and D. W. P. Thomas, Analytical formulation for the shielding effectiveness of enclosures with apertures, IEEE Trans Electromag Compat 40 (1998), 240-247.

2. R. Azaro, S. Caorsi, M. Donelli, and G. L. Gragnani, Evaluation of the effects of an external incident wave on metallic enclosures with rectangular apertures, Microwave Opt Technol Lett 28 (2001), 289-293.

3. A. C. Marvin, J. F. Dawson, S. Ward, L. Dawson, J. Clegg, and A. Weisenfeld, A proposed new definition and measurement of the shielding effect of equipment enclosures, IEEE Trans Electromag Compat 46 (2004), 459-468.

4. Hyun H. Park, Byung W. Kim, Yeon C. Chung, and Joo G. Lee, FDTD analysis of electromagnetic penetration into a rectangular enclosure with multiple rectangular apertures, Microwave Opt Technol Lett 22 (1999), 188-191.

5. F. Olyslager, E. Laermans, D. D. Zutter, S. Criel, R. D. Smedt, N. Lietaert, and A. D. Clercq, Numerical and experimental study of the shielding effectiveness of a Metallic enclosure, IEEE Trans Electromag Compat 41 (1999), 202-212.

6. T. Yamane, A. Nishikata, and Y Shimizu, Resonance suppression of a spherical electromagnetic shielding enclosure by using conductive dielectrics, IEEE Trans Electromag Compat 42 (2000), 441-448.

7. A. Lozano, A. Díaz-Morcillo, J. V. Balbastre, A. B. Calvo, and L. Nuño, Resonance suppression through conductive polymers in an enclosure with PCBs, EMC Europe, Barcelona, Spain, September 2006, 1082-1085.

8. O. M. Ramani, L. Li, Analysis and reduction of electromagnetic field leakage through loaded apertures: a numerical study, Electromagnetics 25 (2005), 679693.

9. A. Lozano, A. Díaz-Morcillo, and J. V. Balbastre, Resonance suppression in enclosures with a metally-lossy dielectric layer by means of genetic algorithms, International Symposium on Electromagnetic Compatibility, Hawaii, USA, July 2007.

10. GoodFellow ${ }^{\circledR}$, Polystyrene conductive sheet, high impact conductive polystyrene, $300 \times 300 \times 1 \mathrm{~mm}^{3}$ and $300 \times 300 \times 3 \mathrm{~mm}^{3}$.

11. Computer Simulation Technology, CST STUDIO SUITE ${ }^{\mathrm{TM}}$ 2008, www.cst.com.

12. M. P. Robinson and J. Clegg, Improved determination of Q-factor and resonant frequency by a quadratic curve-fitting method, IEEE Trans Electromag Compat 47 (2005), 399-402. 


\section{LIST OF CAPTIONS}

Figure 1 Studied enclosure with an external perfect electric conductor (PEC) layer and an inner conductive plastic layer of thickness $t$

Figure 2 Four-layer conductive plastic structure inside a metallic cabinet

Figure 3 Measured SE of the hybrid enclosure (empty and loaded with various thicknesses)

Figure 4 Measured and simulated results with hybrid enclosure $(t=0.8 \mathrm{~cm})$

Figure 5 Resonance suppression minima for a conductivity sweep for the hybrid enclosure $(t=0.2 \mathrm{~cm})$. Simulation

Figure 6 Q-factors for resonance suppression for the hybrid enclosure $(t=0.2 \mathrm{~cm})$. Simulation

Figure 7 Optimized SE results for a $0.2 \mathrm{~cm}$ hybrid structure and a structure removing the outer PEC layer. Simulation 


\section{LIST OF TABLES}

TABLE 1 Measured Q-factors of first two resonances of the enclosure 


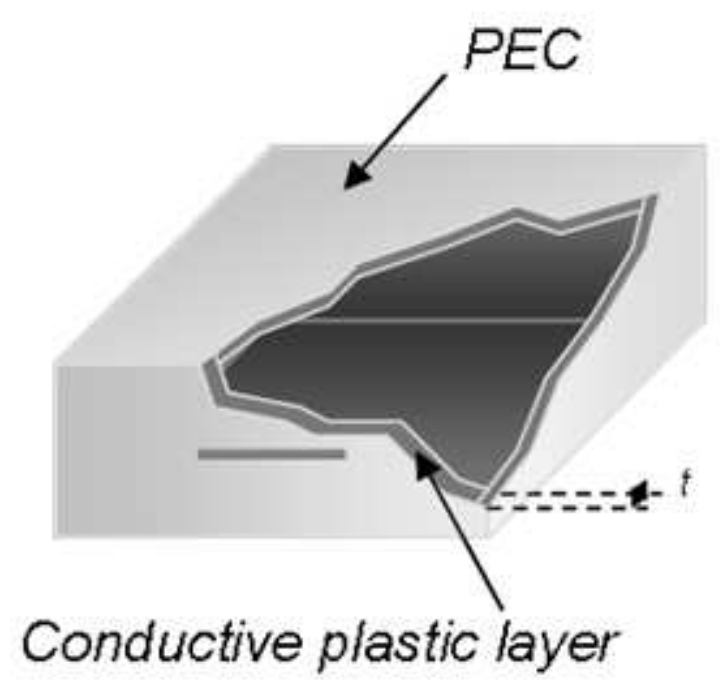

Studied enclosure with an external perfect electric conductor (PEC) layer and an inner conductive plastic layer of thickness $t$

81x83mm (96 x 96 DPI) 


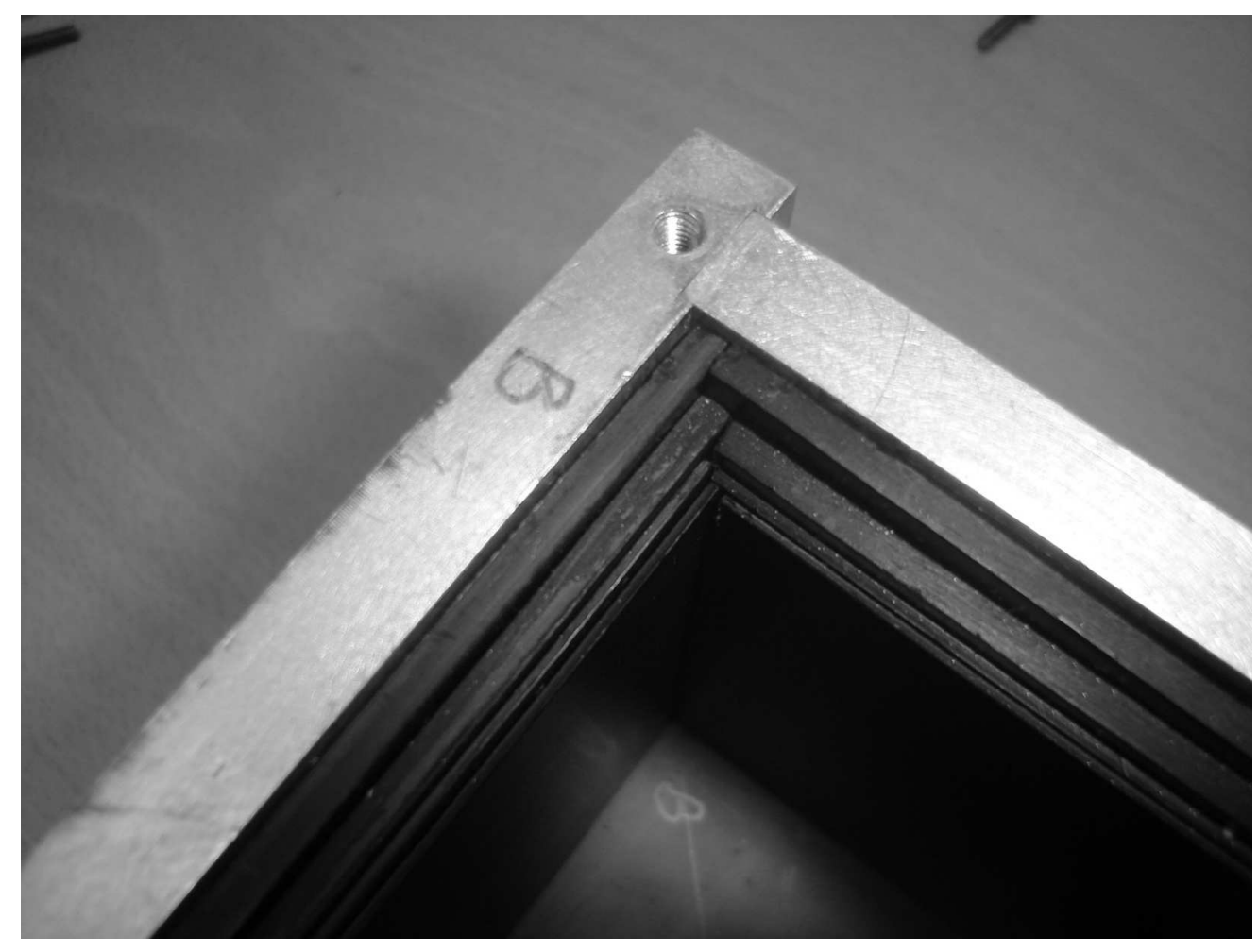

Four-layer conductive plastic structure inside a metallic cabinet $575 \times 431 \mathrm{~mm}(72 \times 72 \mathrm{DPI})$ 


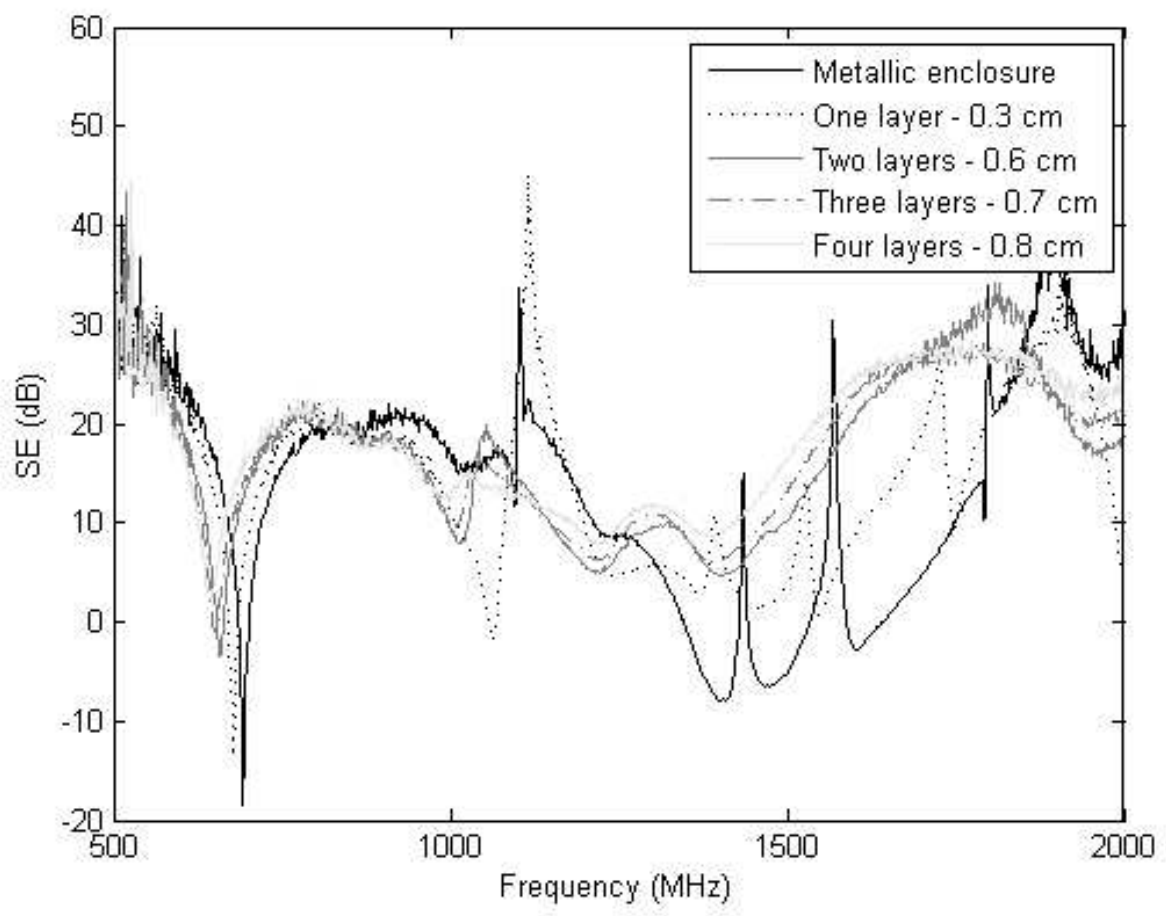

Measured SE of the hybrid enclosure (empty and loaded with various thicknesses) $148 \times 111 \mathrm{~mm}(96 \times 96 \mathrm{DPI})$ 


1
2
3
4
5
6
7
8
9
10
11
12
13
14
15
16
17
18
19
20
21
22
23
24
25
26
27
28
29
30
31
32
33
34
35
36
37
38
39
40
41
42
43
44
45
46
47
48
49
50
51
52
53
54
55
56
57
59
60

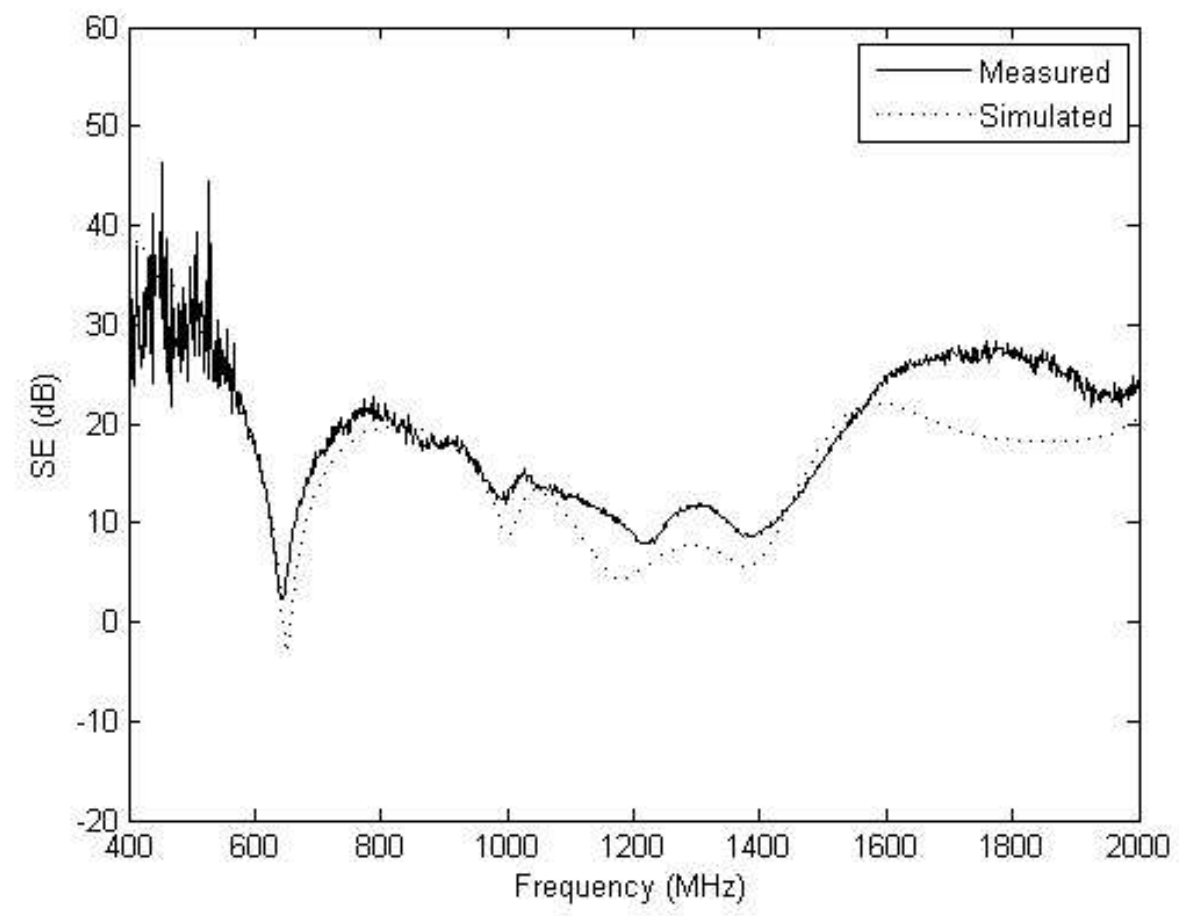

Measured and simulated results with hybrid enclosure $(t=0.8 \mathrm{~cm})$ $148 \times 111 \mathrm{~mm}(96 \times 96 \mathrm{DPI})$ 
Resonance suppression minima for a conductivity sweep for the hybrid enclosure $(t=0.2 \mathrm{~cm})$. Simulation $148 \times 111 \mathrm{~mm}(96 \times 96 \mathrm{DPI})$ 
Optimized SE results for a $0.2 \mathrm{~cm}$ hybrid structure and a structure removing the outer PEC layer. Simulation $148 \times 111 \mathrm{~mm}(96 \times 96$ DPI $)$ 
TABLE 1 Measured Q-factors of first two resonances of the enclosure

\begin{tabular}{ccccc}
\hline$t(\mathrm{~cm})$ & $f_{1}(\mathrm{MHz})$ & $Q_{1}$ & $f_{2}(\mathrm{MHz})$ & $Q_{2}$ \\
\hline Metallic & 693.07 & 418.67 & 1093.79 & 244.61 \\
0.3 & 677.80 & 221.64 & 1062.44 & 73.47 \\
0.6 & 658.24 & 71.12 & 1011.92 & 26.77 \\
0.7 & 651.20 & 52.35 & 1001.99 & 23.77 \\
0.8 & 644.05 & 38.47 & 991.42 & 13.04 \\
\hline
\end{tabular}

